\title{
Uma Abordagem para a Criação e Recomendação de Objetos de Aprendizagem usando um Algoritmo Genético, Tecnologias da Web Semântica e uma Ontologia
}

\author{
Clarivando F. Belizário Júnior, Fabiano A. Dorça
}

Faculdade de Computação - Universidade Federal de Uberlândia (UFU)

Uberlândia - MG - Brasil

clarivando@ufu.br, fabianodor@ufu.br

\begin{abstract}
Resumo. O e-learning é um modelo de ensino eletrônico que pode ser adaptado aos estilos de aprendizagem de cada estudante. A carência de objetos de aprendizagem (OAs) que possam ser recomendados aos alunos é um problema pendente. Outro desafio é a própria recomendação desses OAs. Para solucioná-los, implementa-se uma abordagem que utiliza conteúdo da Wikipédia para a criação de novos recursos educacionais e uma ontologia para a modelagem de estudantes e OAs. Este artigo formaliza o problema de recomendação de OAs como um Problema de Cobertura de Conjuntos, o qual é resolvido por um algoritmo genético (AG). Os resultados dos testes realizados em um protótipo garantem a viabilidade dessa abordagem na solução desses problemas, mostrando que o AG é superior a um algoritmo totalmente aleatório.
\end{abstract}

Abstract. E-learning is an electronic teaching model that can be adapted to student's learning styles. The lack of learning objects (LOs) that can be recommended to students is an open problem. Another challenge is the personalized recommendation of LOs. To solve them, we have implemented an approach that uses Wikipedia content to create new educational resources considering an ontology which models students and LOs. The problem of recommending LOs is formalized, and then a genetic algorithm (GA) is presented to solve it. The tests carried out on a prototype guarantee the viability of this approach to solve these problems, showing that the GA is superior to a random algorithm.

Palavras-chave: Ensino Eletrônico, Web Semântica, Objetos de Aprendizagem

\section{Introdução}

Um importante modelo de ensino eletrônico utilizado atualmente é o e-learning. Nele, os conteúdos educacionais que precisam estar no formato digital para serem armazenados em repositórios e compartilhados entre diversos usuários são designados objetos de aprendizagem (OAs). Cada OA possui dados sobre seu conteúdo denominados metadados, os quais seguem um padrão para facilitar a recuperação do OA. A criação de novos OAs e metadados é geralmente uma tarefa dispendiosa que demanda além do conhecimento do conteúdo, conhecimento técnico.

Consequentemente, os repositórios de OAs não são completos, seja pela falta de conteúdo, seja pelos metadados incompletos. Para solucionar esse problema, neste artigo, propõe-se uma abordagem para a geração automática de conteúdo educacional 
VII Congresso Brasileiro de Informática na Educação (CBIE 2018)

Anais do XXIX Simpósio Brasileiro de Informática na Educação (SBIE 2018)

derivado da Wikipédia. A escolha da Wikipédia deve-se ao fato dela ser uma grande enciclopédia digital com mais de 5 milhões e 708 mil artigos em inglês e mais de 1 milhão de artigos em português (MAIN, 2018). Alguns usuários da comunidade podem editar e marcar artigos com rótulos de qualidade, ajudando os editores a identificarem mais facilmente os artigos que precisam ser melhorados.

Outro desafio é a recomendação de OAs. Esse problema é definido na próxima seção e solucionado por meio de um Algoritmo Genético (WHITLEY, 1994) e tecnologias da Web Semântica (BERNERS-LEE; HENDLER; LASSILA, 2001), doravante WS, que estende a Web tradicional por meio de tecnologias que facilitam a modelagem e manipulação do conhecimento pelas máquinas. As linguagens XML (eXtensible Markup Language), RDF (Resource Definition Framework) e OWL (Web Ontology Language) são utilizadas para representar a informação na WS, e compartilhála entre aplicações, sendo a OWL a linguagem com o maior potencial de representatividade e a XML a linguagem com menor poder de representação da informação. A SWRL (Semantic Web Rule Language) é uma linguagem padrão da WS para a criação de regras que podem ser usadas na inferência de novos conhecimentos.

As principais contribuições deste artigo são:

- Criação de uma ontologia (Gruber, 1993) para a modelagem de OAs conforme o padrão IEEE-LOM (LTSC, 2002) e a modelagem de estudantes, cujos estilos de aprendizagem seguem o modelo proposto por Felder e Silverman (1988). A escolha desse modelo deve-se ao fato dele cobrir mais aspectos psicológicos do que outros modelos (Deborah; Baskaran; Kannan, 2014);

- Criação semiautomática de OAs usando seções da Wikipédia;

- Implementação de uma abordagem que utiliza um Algoritmo Genético para a recomendação automática de OAs considerando sua qualidade, os estilos de aprendizagem do estudante e os parâmetros de filtro definidos pelo usuário.

O problema de recomendação de OAs é descrito na próxima seção. A abordagem proposta para solucioná-lo e os resultados alcançados são apresentados, respectivamente, nas Seções 3 e 4. Na Seção 5, discutem-se os trabalhos relacionados. Por fim, apresentam-se as considerações finais na Seção 6.

\section{Descrição do Problema}

Um dos desafios dos Sistemas Tutores é a recomendação de objetos de aprendizagem que cobrem todos e somente os conceitos que o aluno precisa aprender. No escopo deste trabalho o termo "conceito" é utilizado como sinônimo de "palavra-chave".

Antes da definição formal desse problema, imagine, por exemplo, uma situação em que um aluno precise aprender cinco conceitos, os quais pertencem ao conjunto finito $X=\{\mathrm{C} 1, \mathrm{C} 2, \mathrm{C} 3, \mathrm{C} 4, \mathrm{C} 5\}$. Considere uma coleção de subconjuntos de $X$ dada por $F=\{\mathrm{OA} 1, \mathrm{OA} 2, \mathrm{OA} 3, \mathrm{OA} 4, \mathrm{OA} 5, \mathrm{OA} 6\}$, sendo OA $1=\{\mathrm{C} 1, \mathrm{C} 2\}, \mathrm{OA} 2=\{\mathrm{C} 3\}, \mathrm{OA} 3$ $=\{\mathrm{C} 4, \mathrm{C} 5\}, \mathrm{OA} 4=\{\mathrm{C} 5\}, \mathrm{OA} 5=\{\mathrm{C} 2, \mathrm{C} 3, \mathrm{C} 4, \mathrm{C} 5\}$ e OA6 $=\{\mathrm{C} 2, \mathrm{C} 3\}$. Cada elemento de $F$ é um objeto de aprendizagem que cobre um conjunto de conceitos. O OA6, por exemplo, cobre os conceitos $\mathrm{C} 2$ e $\mathrm{C} 3$. A cada OA de $F$ é atribuído um custo, respeitando a ordem, dado pelo vetor de custos $(3,7,1,1,4,2)$.

O objetivo é encontrar um subconjunto de OAs de $F$, que juntos cobrem todos os elementos (conceitos) de $X$ com custo mínimo. Esse é um problema de Programação 
VII Congresso Brasileiro de Informática na Educação (CBIE 2018)

Anais do XXIX Simpósio Brasileiro de Informática na Educação (SBIE 2018)

Linear Inteira conhecido na literatura como o Problema da Cobertura de Conjuntos (PCC) e de difícil solução, pertencendo à classe NP-Hard (GAREY; JOHNSON, 1979). Para o exemplo anterior, tem-se a solução \{OA1, OA3, OA6\} com custo igual a 6.

Dada uma matriz $a_{i j}$ com $m$ linhas e $n$ colunas, o PCC é um problema de cobertura de linhas por um conjunto de colunas com custo mínimo. Seja $x_{j}=1$, se a coluna $j$ (com custo $c_{j}>0$ ) está na solução, e $x_{j}=0$, caso contrário, então a formulação do PCC é dada por:

$$
\begin{gathered}
\text { Minimize } \sum_{j=1}^{n} c_{j} x_{j} \\
\text { Sujeito } a \sum_{j=1}^{n} a_{i j} x_{j} \geq 1, \quad i=1, \ldots, m \\
x_{j} \in\{0,1\} \quad j=1, \ldots, n
\end{gathered}
$$

A restrição da Eq. (2) garante que cada linha seja coberta por ao menos uma coluna. Note que as colunas da matriz correspondem aos OAs e as linhas aos conceitos. $\mathrm{O}$ custo de escolha de um $O A_{j}$ depende de quão próximo esse $\mathrm{OA}$ é do ideal. $\mathrm{O} O A_{\text {ideal }}$ possui um parâmetro de qualidade e outros parâmetros definidos pelo usuário da ferramenta que implementar a abordagem. Os demais OAs, que possuem esses mesmos parâmetros, são comparados com o $O A_{\text {ideal }}$. Formalmente, seja $\alpha_{i}$ o valor do parâmetro $i$. A função $c_{j}$ da Eq. (1) é calculada para cada par de parâmetros do $O A_{\text {ideal }}$ e um $O A_{j}$ :

$$
c_{j}=\sum_{i=1}^{x}\left|\alpha_{i_{\text {ideal }}}-\alpha_{i_{j}}\right|,
$$

onde $x$ é o número de parâmetros, $\alpha_{i_{i d e a l}}$ é um parâmetro do $O A_{i d e a l}$ e $\alpha_{i_{j}}$ é um parâmetro do $O A_{j}$.

\section{Abordagem Proposta}

A abordagem apresentada a seguir possui três partes principais: o enriquecimento da ontologia com metadados de seções wiki; a recomendação de OAs; e as operações CRUD para criar (Create), ler (Read), atualizar (Update) e deletar (Delete) metadados de OAs e alunos (ver Figura 1). Essas operações podem ser utilizadas pelo professor para inserir também seus próprios OAs na ontologia. O professor precisa apenas setar os parâmetros de busca vinculados aos metadados do padrão IEEE-LOM para a criação de um $O A_{\text {ideal }}$, cujos metadados serão comparados com os metadados dos demais OAs da ontologia para ranqueá-los por ordem de similaridade em relação ao $O A_{\text {ideal }}$. Os campos utilizados e seus respectivos métodos de comparação são:

- Título: os títulos são comparados pela similaridade cosseno;

- Tipo de interatividade: cada termo do vocabulário é mapeado em um valor (active = 0, mixed $=0.5$, expositive $=1$ ) que corresponde ao $\alpha_{i_{j}}$ da Eq. (3);

- Tipo de recurso de aprendizagem: se o $O A_{\text {ideal }}$ e um $O A_{j}$ qualquer são o mesmo tipo de recurso, então, na Eq. (3), $\left|\alpha_{i_{\text {ideal }}}-\alpha_{i_{j}}\right|=0$, caso contrário, $\left|\alpha_{i_{i d e a l}}-\alpha_{i_{j}}\right|=1$;

- Nível de interatividade e densidade semântica: cada termo do vocabulário é mapeado em um valor (very low $=0$; low $=0.25$; medium $=0.5$; high $=0.75$; very high =1) que corresponde ao $\alpha_{i_{j}}$ da Eq. (3); 
VII Congresso Brasileiro de Informática na Educação (CBIE 2018)

Anais do XXIX Simpósio Brasileiro de Informática na Educação (SBIE 2018)

- Grau de dificuldade: cada termo do vocabulário é mapeado em um valor (very easy $=0$, easy $=0.25$, medium $=0.5$, difficult $=0.75$, very difficult $=1$ ) que está em concordância com o parâmetro $\alpha_{i_{j}}$ da Eq. (3).

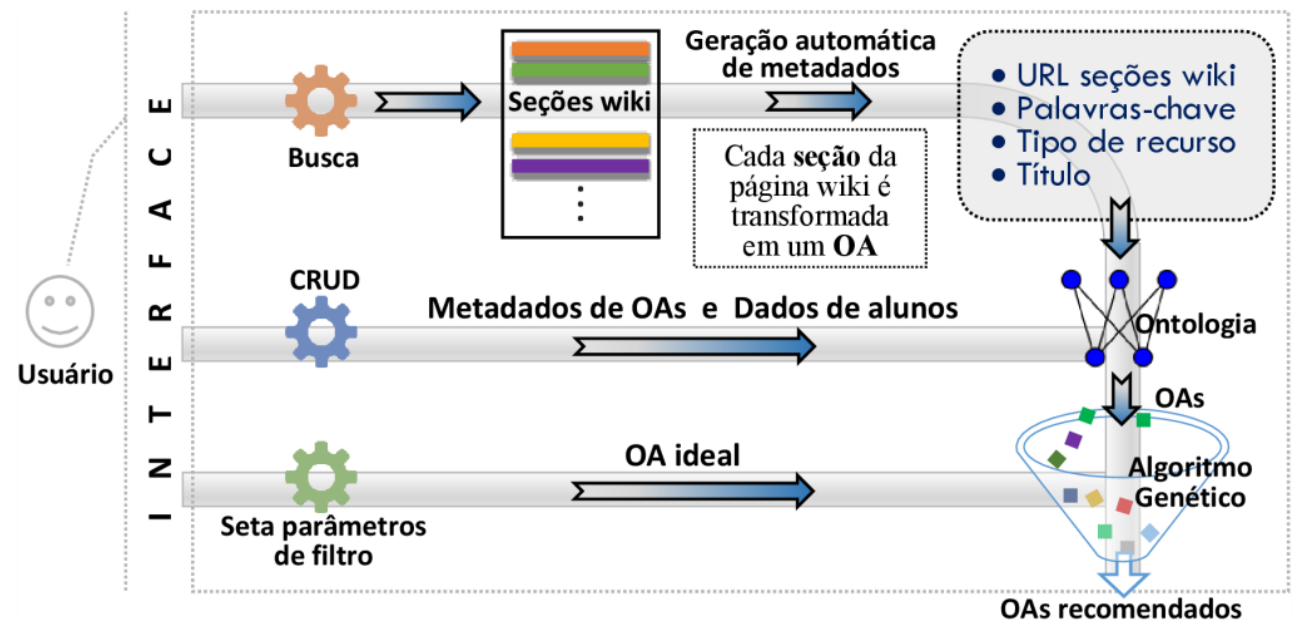

Figura 1: Modelo para a criação e recomendação de objetos de aprendizagem

\subsection{Ontologia}

A ontologia foi construída com a ferramenta Protégé (GENNARI et al., 2003), usando a OWL e a metodologia Ontology Development 101 (NOY; MCGUINNESS, 2001). Os OAs foram modelados conforme o padrão IEEE-LOM e sua extensão Customized Learning Experience Online (CLEO, 2003). As principais classes da ontologia são Aluno, Perfil e ObjetoAprendizagem. O aluno tem um perfil que corresponde ao FSLSM.

Cada objeto de aprendizagem tem as nove categorias do padrão IEEE-LOM. É difícil caracterizar o tipo de recurso de aprendizagem que é uma seção wiki usando apenas o vocabulário do padrão IEEE-LOM. Mas, com a extensão desse vocabulário pelo padrão CLEO, pode-se definir uma seção wiki como: additional resource, definition, introduction ou qualquer outro valor conveniente.

No e-learning, o questionário ILS (Index of Learning Styles) criado por Soloman e Felder (2005) é aplicado tradicionalmente para a identificação dos estilos de aprendizagem do estudante. Dos perfis de estudantes modelados na ontologia infere-se o tipo de recurso de aprendizagem mais adequado para cada aluno. O processo de inferência ocorre por meio de regras SWRL implementadas na ontologia. Neste trabalho, criaram-se 24 regras com base na teoria de Graf, Kinshuk e Ives (2010) que aborda quais OAs devem ser recomendados para cada tipo de perfil de aluno associado ao FSLSM. Kusumawardani, Prakoso e Santosa (2014) exemplificam como essas regras são implementadas.

\subsection{Avaliação da qualidade do conteúdo da Wikipédia}

Warncke-Wang, Cosley e Riedl (2013) utilizaram um classificador baseado em uma árvore de decisão para identificar a qualidade de artigos da Wikipédia. O classificador é capaz de classificar os artigos wiki em sete classes diferentes, quais sejam: Featured Articles (FA), A, Good Articles (GA), B, C, Start e Stub, todas definidas pela própria Wikipédia. 
VII Congresso Brasileiro de Informática na Educação (CBIE 2018)

Anais do XXIX Simpósio Brasileiro de Informática na Educação (SBIE 2018)

No contexto deste artigo, esse classificador é utilizado para avaliar a qualidade de cada seção wiki. Para isso, atribuem-se a cada classe os seguintes valores: FA (1), A (0.85), GA (0.7), B (0.5), C (0.3), Start (0.15) e Stub (0). As seções wiki com o mais alto nível de qualidade (FA) recebem o valor máximo igual a 1 e as seções sem nenhuma qualidade (Stub) recebem nota 0 . A qualidade das seções wiki corresponde a um dos parâmetros definidos pela Eq. (3). Seja $\alpha_{q}$ esse parâmetro de qualidade e, para utilizá-lo na Eq. (3), define-se $\alpha_{q_{\text {ideal }}}=1$ (O $O A_{\text {ideal }}$ pertence à classe FA) e $\alpha_{q_{j}}$ é o valor correspondente à classificação de qualidade recebida pelo $j$-ésimo $\mathrm{OA}$ comparado com o $O A_{\text {ideal }}$.

\subsection{Identificação dos conceitos da seção wiki}

O método utilizado para a extração dos conceitos das seções wiki (ver Figura 2) baseiase na intuição de que o próprio título da seção mais a hierarquia de títulos na qual ela está inserida possam ser usados para a extração dos conceitos que ela trata. Neste caso, após apontar a classe gramatical (POS-tagging) de cada palavra encontrada nessa hierarquia de títulos pelo tokenizer, utiliza-se o chunker para formar noun phrases (NPs). Um NP pode ser qualquer tipo de substantivo, inclusive substantivos intercalados por preposições e caracterizados por adjetivos. Embora alguns substantivos indesejáveis possam ser selecionados como conceitos, quase sempre os títulos das seções contêm os principais conceitos relacionados a elas.

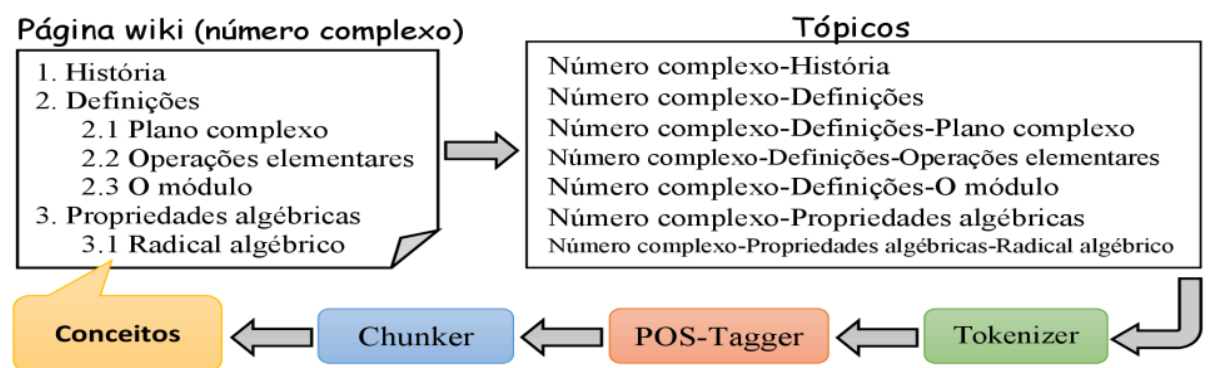

Figura 2: Processamento de um sumário da Wikipédia para a geração de conceitos

A cada seção wiki é atribuído um tópico formado pelo título da seção acrescido dos seus títulos hierarquicamente superiores até o título da página wiki. Cada tópico é utilizado para a extração dos conceitos (palavras-chave) da sua respectiva seção. Note que algumas seções (como a seção "2. Definições”), podem ter ou não algum conteúdo antes da sua primeira subseção. As seções com quase nenhum conteúdo são ignoradas. Sabe-se que o tempo gasto com as técnicas de Processamento da Linguagem Natural tende a aumentar, proporcionalmente, em relação ao tamanho da entrada. A vantagem do método apresentado na Figura 2 é que ele evita o processamento do conteúdo das seções wiki, tornando-se mais rápido.

\subsection{Solução para o problema de recomendação}

Para solucionar o problema de recomendação formalizado na Seção 2, utiliza-se um Algoritmo Genético. O processo de recomendação é individualizado para cada estudante. Para cada aluno atribui-se um $O A_{\text {ideal }}$, cujo parâmetro "tipo de recurso de aprendizagem" é inferido por regras SWRL a partir do perfil do estudante. Esse é um dos parâmetros da função $c_{j}$ definida na Eq. (3) e utilizada no cálculo da fitness. 
VII Congresso Brasileiro de Informática na Educação (CBIE 2018)

Anais do XXIX Simpósio Brasileiro de Informática na Educação (SBIE 2018)

A entrada do AG é a matriz $\alpha_{i_{j}}$ mais o vetor de custos das colunas. As linhas da matriz correspondem aos conceitos do $O A_{\text {ideal }}$ e as colunas correspondem aos OAs da ontologia que cobrem ao menos uma linha (conceito). Cada OA de $\alpha_{i_{j}}$ é indexado univocamente por valores inteiros utilizados na composição dos cromossomos. Cada indivíduo é estruturado por um vetor de inteiros (começando do zero) com $k$ posições, em que $k$ é o número de conceitos do $O A_{\text {ideal }}$. A Figura 3 contém três exemplos de indivíduos associados a uma matriz com 8 linhas (conceitos) e 5 colunas (OAs). A função fitness $F$, de um indivíduo $i$, está diretamente associada ao valor da sua função objetivo (Eq. (1)) e é calculada por:

$$
F_{i}=\sum_{j=1}^{n} c_{j} s_{i j}
$$

onde $c_{j}$ é o custo da coluna $j$ e $s_{i j}$ é 1 se a coluna $j$ pertence ao $i$-ésimo indivíduo, e 0 , caso contrário.

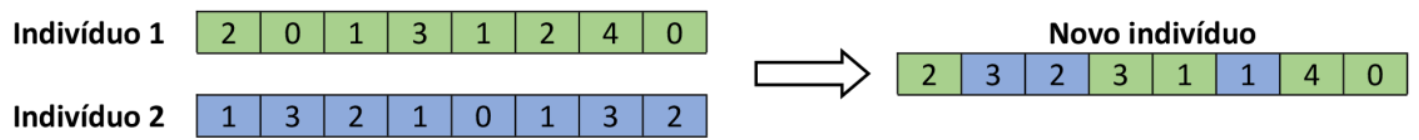

Figura 3: Crossover incremental

Neste trabalho, aplica-se o Método do Torneio para a seleção de quatro indivíduos. São quatro torneios, cada qual formado por dois indivíduos escolhidos aleatoriamente. Seleciona-se o indivíduo mais apto de cada torneio. Esses indivíduos formam duas duplas. Para cada dupla aplica-se o cruzamento incremental para a geração de um novo indivíduo (ver Figura 3). O crossover de dois indivíduos 1 e 2, com fitness, respectivamente, $F_{1}$ e $F_{2}$, gera um filho do qual cada gene é preenchido com o gene de mesma posição de um dos indivíduos pais. $\mathrm{O}$ gene do indivíduo 1 é escolhido com uma probabilidade $P=F_{2} /\left(F_{1}+F_{2}\right)$. Consequentemente, o gene do indivíduo 2 é escolhido com probabilidade $1-P$. Assim, a cada geração, dois novos indivíduos são criados. A mutação é aplicada com uma probabilidade de $10 \%$ para substituir um OA (valor inteiro) escolhido aleatoriamente por outro. Após a mutação, executa-se um procedimento para tornar cada indivíduo viável, garantido que as colunas do indivíduo cubram seus respectivos conceitos. O método de substituição utilizado considera a fitness média da população para identificar os indivíduos menos aptos (com fitness acima da média). Dois desses indivíduos são escolhidos aleatoriamente para serem substituídos pelos dois novos indivíduos.

\section{Experimentos e Resultados}

Implementou-se um protótipo em Python para testar a eficiência da abordagem. Em todos os testes, executa-se o AG apresentado na Seção 3.4 com uma população de 100 indivíduos. O AG implementado é comparado com um algoritmo randômico, no qual cada novo indivíduo é uma solução gerada aleatoriamente. Ambos algoritmos foram executados cinco vezes, tendo como entrada uma matriz de 200 linhas e 1000 colunas mais seu vetor de custos derivados da instância 4.10 do benchmark OR-Library ${ }^{1}$, o qual possui instâncias grandes do PCC disponíveis para testes. Na Figura 4, compara-se a

\footnotetext{
${ }^{1}$ Disponível em: http://people.brunel.ac.uk/ mastjjb/jeb/orlib/scpinfo.html
} 
VII Congresso Brasileiro de Informática na Educação (CBIE 2018)

Anais do XXIX Simpósio Brasileiro de Informática na Educação (SBIE 2018)

fitness média das 5 execuções de cada algoritmo, considerando 5000 gerações com dois novos indivíduos criados em cada uma. A solução ótima para essa instância é 514. O AG obtém na média uma fitness de 515.8, um valor próximo do ótimo e bem melhor que o algoritmo randômico.

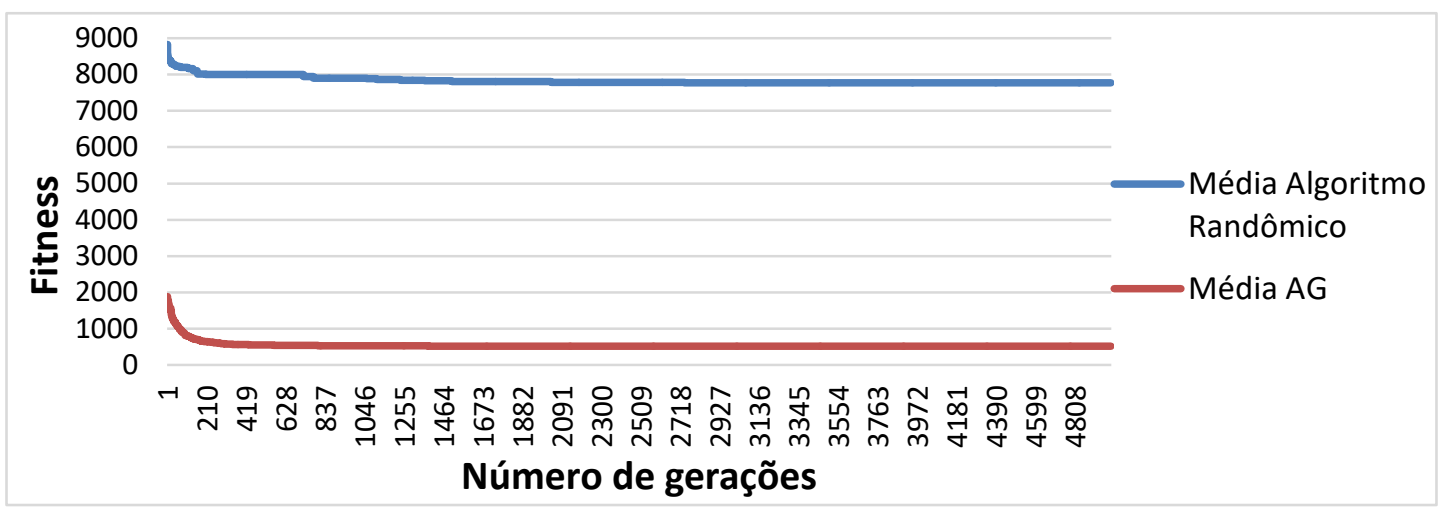

Figura 4: Comparação dos desempenhos dos algoritmos genético e randômico

Realizaram-se mais dois testes com o AG apresentado na Seção 3.4 para testar o funcionamento de toda a abordagem. Para isso, a ontologia foi enriquecida com metadados (Quadro 1) de 49 OAs correspondentes a 49 seções de 4 páginas da Wikipédia resultantes da busca do usuário por conteúdo associado aos conceitos mitose, meiose, fissão binária e reprodução.

Quadro 1: Preenchimento dos campos dos OAs para a execução dos testes

\begin{tabular}{|c|c|c|}
\hline Atributos & $\boldsymbol{O A}_{\text {ideal }}$ & $\begin{array}{c}\text { 49 OAs disponíveis para } \\
\text { recomendação }\end{array}$ \\
\hline $\begin{array}{c}\text { Palavras-chave } \\
\text { (campo 1.5-LOM) }\end{array}$ & $\begin{array}{c}\text { Reprodução, Meiose, Prófase, } \\
\text { Divisão Equacional, Metáfase }\end{array}$ & $\begin{array}{c}\text { Método de geração automática de } \\
\text { palavras-chave apresentado na Fig. } 2 .\end{array}$ \\
\hline $\begin{array}{c}\text { Título (campo } \\
\text { 1.2-LOM) }\end{array}$ & Divisão celular & $\begin{array}{c}\text { Preenchido com o título da seção wiki } \\
\text { correspondente ao OA. }\end{array}$ \\
\hline $\begin{array}{c}\text { Tipo de recurso } \\
\text { (campo 5.2-LOM) }\end{array}$ & Introduction & Preenchimento aleatório \\
\hline Qualidade & $\alpha_{q_{i d e a l}}=1$ & $\alpha_{q_{j}}$ aleatório; $0 \leq \alpha_{q_{j}} \leq 1$ \\
\hline Dificuldade & easy $\left(\alpha_{i_{i d e a l}}=0.25\right)$ & Preenchimento aleatório \\
\hline $\begin{array}{c}\text { URL da seção } \\
\text { (campo 1.1.2-LOM) }\end{array}$ & (Não se aplica) & $\begin{array}{c}\text { Preenchido com o URL da página } \\
\text { concatenado com o título da seção }\end{array}$ \\
\hline
\end{tabular}

Uma questão que permanece em aberto é definir que tipo de recurso de aprendizagem são as seções wiki. Em cenários reais, os tipos de recursos são diversificados, e para simular esse cenário, considera-se, "apenas para a execução dos testes", que as seções wiki possam assumir qualquer tipo de recurso. Assim, para testar a qualidade da abordagem em relação ao "tipo de recurso de aprendizagem", esse foi escolhido aleatoriamente dentre os 15 tipos definidos pelo padrão IEEE-LOM mais os tipos definition, example, introduction, additional resource e assessment definidos pelo padrão CLEO. O campo qualidade foi preenchido aleatoriamente com valores reais de 0 a 1 (o classificador apresentado na Seção 3.2 não foi implementado no protótipo, mas isso não compromete os resultados).

A entrada do AG nos dois testes corresponde a uma matriz com 3 linhas e 47 OAs. O conceito "Prófase" é coberto apenas pelo OA10 e o conceito "Metáfase" é 
VII Congresso Brasileiro de Informática na Educação (CBIE 2018)

Anais do XXIX Simpósio Brasileiro de Informática na Educação (SBIE 2018)

coberto apenas pelo OA12. Assim, esses OAs fazem, necessariamente, parte da solução e são, portanto, junto com todos os conceitos que eles cobrem, eliminados da entrada do AG. O custo de cada coluna é calculado pela Eq. (3), comparando-se a similaridade dos seus parâmetros título, tipo de recurso de aprendizagem, qualidade e grau de dificuldade em relação ao $O A_{\text {ideal }}$. Na Tabela 1 , comparam-se as três melhores soluções do teste 1 , que não considera o parâmetro $\alpha_{q_{j}}$, com o teste 2 que o utiliza.

Tabela 1: Influência do parâmetro de qualidade na solução do problema

\begin{tabular}{|c|c|c|c|c|c|}
\hline \multicolumn{3}{|c|}{ Teste 1: Parâmetro $\alpha_{q_{j}}$ não utilizado } & \multicolumn{3}{|c|}{ Teste 2: Parâmetro $\alpha_{q_{j}}$ utilizado $\left(\alpha_{q_{\text {ideal }}}=1\right)$} \\
\hline Soluções & $\alpha_{q_{j}}$ & Fitness & Soluções & $\alpha_{q_{j}}$ & Fitness \\
\hline OA12 exam & 0.9153 & \multirow{4}{*}{0,7306} & OA12 exam & 0.9153 & \multirow{4}{*}{0,8048} \\
\hline OA10 introduction & 0.6237 & & OA10 introduction & 0.6237 & \\
\hline OA40 introduction & 0.3626 & & OA40 introduction & 0.3626 & \\
\hline OA32 introduction & 0.4977 & & OA29 introduction & 0.7854 & \\
\hline OA12 exam & 0.9153 & \multirow{4}{*}{0,7931} & OA12 exam & 0.9153 & \multirow{4}{*}{0,8124} \\
\hline OA10 introduction & 0.6237 & & OA10 introduction & 0.6237 & \\
\hline OA40 introduction & 0.3626 & & OA40 introduction & 0.3626 & \\
\hline OA29 introduction & 0.7854 & & OA32 introduction & 0.4977 & \\
\hline OA12 exam & 0.9153 & \multirow{4}{*}{0,8556} & OA12 exam & 0.9153 & \multirow{4}{*}{0,8825} \\
\hline OA10 introduction & 0.6237 & & OA10 introduction & 0.6237 & \\
\hline OA41 introduction & 0.0478 & & OA46 introduction & 0.4743 & \\
\hline OA32 introduction & 0.4977 & & OA29 introduction & 0.7854 & \\
\hline
\end{tabular}

Note que a utilização desse parâmetro resulta na seleção de OAs que possuem um valor $\alpha_{q_{j}}$ alto. Contudo, os OAs recomendados também dependem dos pesos (que variam de 0 a 1) atribuídos a cada parâmetro. Todos os parâmetros dos testes anteriores possuem peso igual a 1 . $\mathrm{Na}$ análise dos pesos em diversos outros testes realizados, constatou-se que os OAs das melhores soluções tendem a ser mais similares ao OA ideal em relação aos parâmetros que possuem um peso maior que os demais parâmetros.

\section{Trabalhos Relacionados}

Nesta seção, apresentam-se os trabalhos relacionados ao uso de ontologias na recomendação de OAs e trabalhos que utilizaram a Wikipédia na aprendizagem. A literatura correlata carece de trabalhos que fazem o reúso de conteúdo web na criação de objetos de aprendizagem explorando tecnologias da WS e ontologias. Contudo, há trabalhos que implementam parte desse processo. No Quadro 2, apresentam-se quatro desses trabalhos relacionando-os com a abordagem apresentada neste artigo.

Gaeta et al. (2013) utilizaram ontologias para modelar domínios de conhecimento e um algoritmo guloso no processo de recomendação de OAs. Jácome Júnior, Mendes Neto e Da Silva (2012), por sua vez, utilizaram ontologias para representar os perfis estático e dinâmico dos alunos, as quais são combinadas com agentes de software e um AG para a recomendação de OAs de maneira autônoma.

Em relação a Wikipédia, há muitos trabalhos da literatura correlata que a utilizaram na aprendizagem. Para citar um exemplo, Limongelli, Gasparetti e Sciarrone (2015) desenvolveram um módulo de um sistema para auxiliar professores na construção de cursos que contêm apenas páginas da Wikipédia. O processo de recomendação das páginas considera as filtragens por conteúdo e social, na qual o professor pode se beneficiar das escolhas já realizadas por outros usuários que possuem um estilo de ensino parecido com o seu. 
VII Congresso Brasileiro de Informática na Educação (CBIE 2018)

Anais do XXIX Simpósio Brasileiro de Informática na Educação (SBIE 2018)

Quadro 2: Comparação da literatura correlata com a proposta deste trabalho

\begin{tabular}{|c|c|c|c|c|c|}
\hline $\begin{array}{c}\text { Literatura } \\
\text { correlata }\end{array}$ & $\begin{array}{c}\text { Criação } \\
\text { de OAs }\end{array}$ & $\begin{array}{c}\text { Padrões de } \\
\text { metadados }\end{array}$ & $\begin{array}{c}\text { Reúso de } \\
\text { conteúdo } \\
\text { web }\end{array}$ & $\begin{array}{c}\text { Ontologia e/ou } \\
\text { tecnologias da } \\
\text { WS }\end{array}$ & $\begin{array}{c}\text { Técnica de } \\
\text { recomendação } \\
\text { de OAs }\end{array}$ \\
\hline $\begin{array}{c}\text { Menolli, Malucelli } \\
\text { e Reinehr (2011) }\end{array}$ & Sim & IEEE-LOM & Sim & Não & Não \\
\hline $\begin{array}{c}\text { Jácome Júnior, } \\
\text { Mendes Neto e Da } \\
\text { Silva (2012) }\end{array}$ & Não & SCORM & Não & Sim & $\begin{array}{c}\text { Algoritmo } \\
\text { Genético }\end{array}$ \\
\hline Gaeta et al. (2013) & Não & IEEE-LOM & Não & Sim & $\begin{array}{c}\text { Algoritmo } \\
\text { Guloso }\end{array}$ \\
\hline $\begin{array}{c}\text { Limongelli, } \\
\text { Gasparetti e } \\
\text { Sciarrone (2015) }\end{array}$ & Não & Não & Sim & Não & $\begin{array}{c}\text { Filtragens por } \\
\text { conteúdo e } \\
\text { colaborativa }\end{array}$ \\
\hline Nossa abordagem & Sim & $\begin{array}{c}\text { IEEE-LOM } \\
\text { CLEO }\end{array}$ & Sim & Sim & $\begin{array}{c}\text { Algoritmo } \\
\text { Genético }\end{array}$ \\
\hline
\end{tabular}

Por outro lado, na realização desta pesquisa, encontrou-se apenas um trabalho no qual os autores propuseram uma abordagem para gerar OAs a partir de páginas wiki, utilizando o padrão IEEE-LOM. Menolli, Malucelli e Reinehr (2011) utilizaram técnicas de mineração de texto nas páginas wiki para a geração de alguns metadados, os quais são revisados por um especialista humano, que pode criar os demais metadados manualmente. Os autores não utilizaram ontologias e tecnologias da WS.

\section{Considerações Finais}

Dois desafios no contexto do e-learning são a carência de recursos educacionais e a recomendação deles conforme o estilo de aprendizagem de cada estudante. Para lidar com esses problemas, neste trabalho, apresenta-se uma abordagem para a recomendação personalizada de OAs usando conteúdo wiki e uma ontologia. O AG implementado resolve o problema da recomendação de OAs retornando soluções que possuem elevada qualidade quando o parâmetro $\alpha_{q_{j}}$ é considerado. Isso demonstra a viabilidade dessa abordagem na recomendação de OAs derivados de seções wiki.

Algumas questões inerentes a este trabalho são: "O conteúdo da Wikipédia é apropriado para todos os níveis de ensino?" e "Qual a relação (diferenças e similaridades) entre palavras-chave e conceitos?". Em trabalhos futuros, visa-se responder a questões como essas e implantar a abordagem apresentada em um ambiente virtual de aprendizagem, suprindo a falta de recursos educacionais e recomendando os OAs mais adequados a cada perfil de aluno.

\section{Agradecimentos}

Os autores agradecem ao CNPq e à Pró-Reitoria de Pesquisa e Pós-Graduação por todo o suporte prestado no desenvolvimento deste trabalho.

\section{Referências}

BERNERS-LEE, T.; HENDLER, J.; LASSILA, O. The semantic web. Scientific american, JSTOR, v. 284, n. 5, p. 34-43, 2001.

CLEO LAB. PARTNERS. CLEO Extensions to the IEEE Learning Object Metadata. Washington, USA, 2003. 65 p. 
VII Congresso Brasileiro de Informática na Educação (CBIE 2018)

Anais do XXIX Simpósio Brasileiro de Informática na Educação (SBIE 2018)

DEBORAH, L. J.; BASKARAN, R.; KANNAN, A. Learning styles assessment and theoretical origin in an e-learning scenario: a survey. Artificial Intelligence Review, Springer, v. 42, n. 4, p. 801-819, 2014.

FELDER, R. M.; SILVERMAN, L. K. Learning and teaching styles in engineering education. Engineering education, v. 78, n. 7, p. 674-681, 1988.

GAETA, M. et al. An approach to personalized e-learning. JEIC, v. 11, n. 1, 2013.

GAREY, M. R.; JOHNSON, D. S. Computers and intractability: A guide to the theory of np-completeness. New York: W. H. Freeman \& Co., 1979. 340 p.

GENNARI, J. H. et al. The evolution of protÉgé: An environment for knowledge-based systems development. IJHCS, Elsevier, v. 58, n. 1, p. 89-123, 2003.

GRAF, S.; KINSHUK; IVES, C. A flexible mechanism for providing adaptivity based on learning styles in learning management systems. In: ICALT, 10., 2010, Washington, USA. Proceedings... Washington: IEEE, 2010. p. 30-34.

GRUBER, T. R. A translation approach to portable ontology specifications. Knowledge acquisition, Elsevier, v. 5, n. 2, p. 199-220, 1993.

JÁCOME JÚNIOR, L.; MENDES NETO, F. M.; DA SILVA, L. C. N. Uma abordagem baseada em algoritmo genético para recomendação de objetos de aprendizagem sensível ao contexto do estudante. In: SBIE, 23., 2012, RJ. Anais... RJ: SBC, 2012.

KUSUMAWARDANI, S. S.; PRAKOSO, R. S.; SANTOSA, P. I. Using ontology for providing content recommendation based on learning styles inside e-learning. In: AIMS, 2., 2014, Madrid. Proceedings... Washington: IEEE, 2014. p. 276-281.

LEARNING TECHNOLOGY STANDARDS COMMITTEE. IEEE 1484.12.1: Draft standard for learning object metadata. Nova York, 2002. $44 \mathrm{p}$.

LIMONGELLI, C.; GASPARETTI, F.; SCIARRONE, F. Wiki course builder: A system for retrieving and sequencing didactic materials from wikipedia. In: ITHET, 14., 2015, Lisbon. Proceedings... Washington: IEEE Computer Society, 2015.

MAIN page. In: Wikipedia: the free encyclopedia. Disponível em: <https://en.wikipedia.org>. Acesso em: 30 ago. 2018.

MENOLLI, A.; MALUCELLI, A.; REINEHR, S. Criaçao semi-automatica de objetos de aprendizagem a partir de conteúdos da wiki. In: SBIE, 22., 2011, Aracaju, Sergipe. Anais... Porto Alegre, RS: SBC, 2011. p. 214-223.

NOY, N. F.; MCGUINNESS, D. L. Ontology Development 101: A Guide to Creating Your First Ontology. 2001.

SOLOMAN, B. A.; FELDER, R. M. Index of learning styles questionnaire. 2005. NC State University. Disponível em: 〈https://www.webtools.ncsu.edu/learningstyles/>.

WARNCKE-WANG, M.; COSLEY, D.; RIEDL, J. Tell me more: An actionable quality model for wikipedia. In: OPENSYM, 9., 2013, Hong Kong. Proceedings... NY: ACM, 2013.

WHITLEY, D. A genetic algorithm tutorial. Statistics and computing, Springer, v. 4, n. 2, p. 65-85, 1994. 